\title{
A survey assessing methods of maintaining denture hygiene among denture wearers
}

Ghimire $\mathrm{B}^{1}$, Dhital $\mathrm{S}^{2}$

${ }^{1}$ Assistant Professor, Department of Prosthodontics, Kantipur Dental College and Hospital, Basundhara, Kathmandu, Nepal.

${ }^{2}$ Lecturer, Department of Prosthodontics, Kantipur Dental College and Hospital, Basundhara, Kathmandu, Nepal.

\begin{abstract}
Introduction: This study aimed to investigate the denture hygiene habits among old age denture wearers. The purpose of the study was to explain about the methods of maintaining denture hygiene among denture wearers.

Methods: The present cross-sectional survey was conducted among denture wearer patients during follow-up appointment, using a self-administered structured questionnaire.

Results: The study sample consisted of a total of 324 participants; out of them, $211(65.1 \%)$ were males and $113(34.9 \%)$ were females. Regarding the method of maintaining denture cleanliness, most of the patients cleaned it with water only $(57.1 \%)$, whereas patients who cleaned with brush and paste were less $(35.8 \%)$ and very few cleaned it with a combination of water, brush and toothpaste $(7.1 \%)$. Conclusion: Majority of the patients cleaned their denture at least once daily and were provided with proper instructions from their dentist to maintain denture hygiene.
\end{abstract}

Key words: Cleaning habits, Denture hygiene, Edentulism, Elderly patients

\section{Introduction}

$\mathrm{D}$ ental diseases like dental caries, periodontal disease, dentoalveolar trauma are the major causes for the loss of natural dentition. Removable partial dentures and complete dentures are commonly used rehabilitation modes for partially and completely edentulous arches respectively. ${ }^{1}$

Acrylic is the most commonly used material in denture fabrication, which contains micro porous surfaces that provide environments to support microorganisms. It is vital that patients do not neglect the daily cleaning of their dentures as poor hygiene may lead to halitosis, staining

\section{Conflict of Interest: No}

\section{*Corresponding Author}

Dr. Barsha Ghimire, Assistant Professor

Department of Prosthodontics, Kantipur Dental

College and Hospital,Kathmandu, Nepal

E-mail: b28barsha@gmail.com of the dentures and acrylic teeth, irritation to tissues, calculus deposition on teeth as well as yeast infections of the oral cavity. ${ }^{2}$

Patients should be instructed to rinse their mouth and dentures after meals whenever possible. ${ }^{3,4}$ The intra mucosal surfaces of the denture bases can be the favorable place for the formation of new reservoir of microorganisms. However, it has been observed that the majority of denture wearers do not pay necessary attention to the cleanliness. ${ }^{5}$ The mechanical approach to clean dentures include brushing it with either hot or cold water. Mechanical method is mostly accompanied with a chemical one to attain effective cleaning, maintenance and infection control. ${ }^{6}$ Dental professionals must have proper information of denture cleansing strategies to maximize the services offered to their denture patients before using the dentures. ${ }^{7}$

Hence this study aimed to investigate the denture hygiene habits among denture wearers. 
The purpose of the study was to explain denture wearers about the methods of maintaining denture hygiene.

\section{Methods}

Thepresentcross-sectional surveywasconducted among denture patients during follow-up visit in the Department of Prosthodontics, Kantipur Dental College and Hospital, Basundhara, Kathmandu, Nepal from July 2020 to December 2020. The ethical clearance was obtained from Institutional Review Committee, Kantipur Dental College (IRC-KDC) to conduct the study. The purpose of the survey was explained to the subjects and written consent was obtained. The patients wearing complete denture, removable partial denture or both were included in the study. However, new denture wearers were excluded from the study.

Pilot testing of the questionnaire was conducted among a group of 30 patients to test reliability and validity of the questionnaire. The participants were asked to fill up the anonymous, self-administered questionnaire. The questionnaire contained demographic information such as age, sex, duration of denture wear, and questions related to the attitude of denture hygiene habits, frequency of cleaning, and nocturnal denture wearing habits. Sample size was calculated using the following formula using the prevalence of edentulous patient from the previous study.

Sample size $(N)=\underline{Z^{2}}=\underline{p q}$

$$
\mathrm{e}^{2}
$$

Data were analyzed using SPSS version 15.0. Descriptive statistics were obtained, and frequency distribution, means, and standard deviation were calculated. Simple descriptive statistics were used together with Chi-square test at $P<0.05$ to evaluate the relationship between age, gender and prosthetic treatment.

\section{Results}

The study sample consisted of a total of 324 participants; out of them, 211 (65.1\%) were males and 113 (34.9\%) were females. They were further categorized according to age groups as 30-39 years (Group I), 40-49 years (Group II), 50-59 years (Group III), 60-69 years (group IV) and above 70 (Group V). With respect to age distribution of the sample, 103 belonged to the age group of 30-39 years, 70 subjects were of 40-49 years of age, and 130 were 50-59 years of age, 20 were 60-69 years of age and 1 was above 70 .

Regarding the method of maintaining denture cleanliness, most of the patients cleaned it with water only $(57.1 \%)$, whereas patients who cleaned with brush and paste were less (35.8\%) and very few cleaned it with a combination of water, brush and toothpaste (7.1\%).

Regarding the frequency of denture cleaning $268(82.7 \%)$ cleaned the denture once daily, $10(3.1 \%)$ cleaned the denture twice daily, only $46(14.2 \%)$ cleaned their denture after each meal.

There was no relationship between the age and denture wearing while sleeping. The age of 30-39 years wear denture at night-time (59.2\%).

Also, regarding the night wearing of the denture patient-wearing denture during sleeping was less $25.6 \%$ compared to the patients not wearing denture $74.4 \%$.

Among 324 participants 210(64.8\%) received instructions from dentists regarding denture cleaning.

\begin{tabular}{|c|c|c|c|c|c|}
\hline & & Frequency & Percent & Valid Percent & Cumulative Percent \\
\hline \multirow{3}{*}{ Valid } & Yes & 83 & 25.6 & 25.6 & 25.6 \\
\hline & No & 241 & 74.4 & 74.4 & 100.0 \\
\hline & Total & 324 & 100.0 & 100.0 & \\
\hline
\end{tabular}




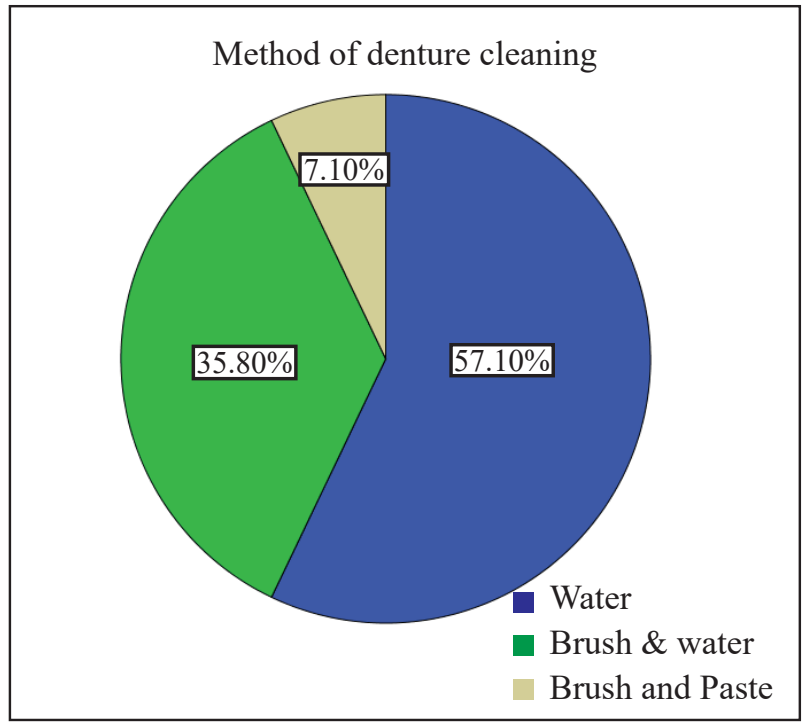

Figure 1: Method of denture cleaning

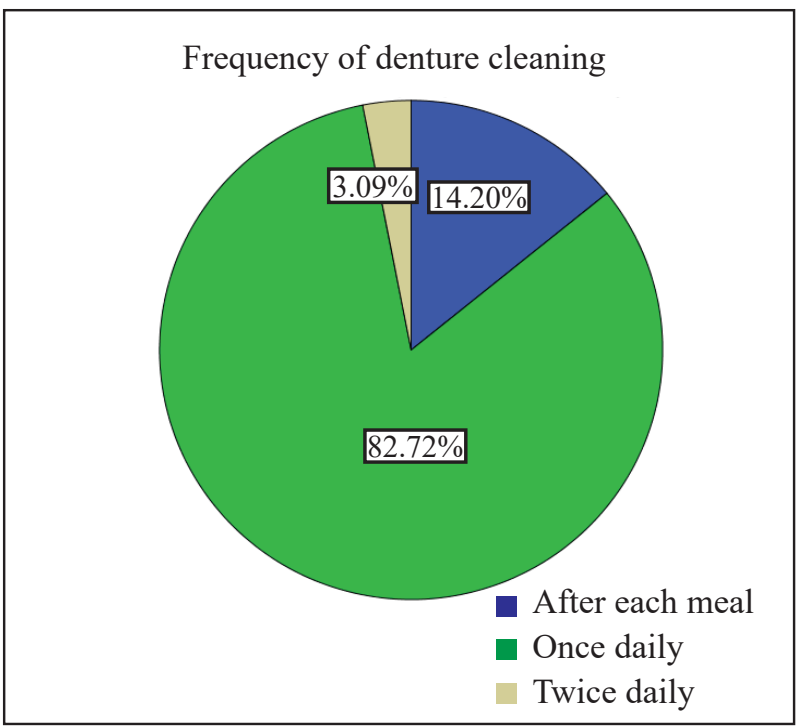

Figure 2: Frequency of denture cleaning

dirty dentures. Majority of the patients receive proper instructions regarding the ways in which they can clean their dentures, yet the patients sometimes fail to do so efficiently and regularly. For this reason, dentists should also provide them with thorough knowledge regarding the adverse effects of wearing unclean dentures.

The majority of patients exhibited limited knowledge regarding the care and cleaning of denture. Most of the patients used water only to clean the denture and did not use any denture cleansers. So the dentists should instruct their patients a combination of brushing technique and soaking in cleansing solutions. Older prosthesis tend to be more dirty causing oral lesions such as oral candidiasis and denture stomatitis. This could be due to many microorganisms that could have collected on the denture surface over the years. They may even have deposits present on the denture due to insufficient cleaning of the denture.

The present study had a few limitations. The technical quality of the prosthesis was not assessed in any way. Furthermore, patient satisfaction was also not taken into consideration as in article given by Waseem et al. ${ }^{10}$ 


\section{Conclusions}

From the study, it was concluded that majority of the patients cleaned their denture at least once daily and were provided with proper instructions from their dentist to maintain the dentures. Regular visits to the dentists for the assessment of the status of denture hygiene and maintenance instructions will be fruitful for the patients to get better use and maintenance of their denture and improve their oral health.

Acknowledgement: We would like to thank all the participants of this study.

\section{References}

1. SahaA, Dutta $S$, Varghese Rk, Kharsan V, Agrawal A. A survey assessing modes of maintaining denture hygiene among elderly patients. J Int Soc Prevent Comm Dent 2014;4(3).

2. Kalaignan SP. "Level Of Denture Hygiene Education And The Quality Of Denture Fabricated By Dental Students". Acta Scientific Dental Sciences 1.1 (2017): 04-08.

3. Kanli A, Demirel F, Sezgin Y. Oral Candidosis, Denture Cleanliness And Hygiene Habits In An Elderly Population. Aging ClinExp Res 2005; 17:502-7.
4. Polyzois Gl. Denture Cleansing Habits. A Survey. Aust Dent J 1983;28:171-3.

5. Lerra S, Khajuria N. A clinical survey of 250 cases of denture hygiene habits and oral tissue conditions of complete denture wearers. Intl journal of App Dent Sci 2017;3(4:252-254.0

6. Harikrishnan R, Ganapathy D. A survey on assessing the denture hygiene awareness among elderly patients. Drug Invent Today|Vol 11.Iss 11.2019.

7. Akram M, Iqbal Q, Naeem S, Denture Hygiene Knowledge And Practices Among Complete Denture Wearers Seen At Dental Hospital. Jnda|Vol 19 No.2.

8. Peracini A, Andrade Im, ParanhosHfo, Souza Rf, Behaviours And Hygiene Habits Of Complete Denture Wearers, Braz Dent J 21 (3)2010.

9. Jandial S, Kotwal B, Sharma S, Mahajan N, Kharyal S, Chadda AS, Evaluation of denture hygiene maintenance among denture wearers in jammupopulation.Int J Prevent Public Health Sci 2017;2(5):27-29.

10. WaseemAUl, Magray IA, Tahleel, Bashi A. A survey of the prosthetic status and post-treatment satisfactionof patients among Kashmiri population visiting Government Dental College and Hospital Srinagar.Intl Jrnl of AdvResearch, Ideas and Innovations in Technology. 2019, Vol 5, Iss 3 . 